\title{
Approximation properties of solutions of a mean value type functional inequalities
}

\author{
Ginkyu Choi ${ }^{a}$, Soon-Mo Jung ${ }^{\mathrm{b}, *}$, Yang-Hi Lee ${ }^{\mathrm{c}}$ \\ ${ }^{a}$ Department of Electronic and Electrical Engineering, College of Science and Technology, Hongik University, 30016 Sejong, Republic of \\ Korea. \\ ${ }^{b}$ Mathematics Section, College of Science and Technology, Hongik University, 30016 Sejong, Republic of Korea. \\ ${ }^{c}$ Department of Mathematics Education, Gongju National University of Education, Gongju 32553, Republic of Korea.
}

Communicated by K. Cieplinski

\begin{abstract}
We will prove the generalized Hyers-Ulam stability theorems of a mean value type functional equation, namely

$$
f(x)-g(y)=(x-y) h(s x+s y),
$$

which arises from the mean value theorem. As an application of our results, we introduce a characterization of quadratic polynomials. (C)2017 All rights reserved.

Keywords: Hyers-Ulam stability, generalized Hyers-Ulam stability, mean value type functional equation, characterization of quadratic polynomials.

2010 MSC: 39B82, 39B62, 39B52, 46J99.
\end{abstract}

\section{Introduction}

The stability problem of functional equations seems to be first formulated by Ulam in 1940. Indeed he discussed a number of important unsolved problems (see [17]):

Let $\mathrm{G}_{1}$ be a group and let $\mathrm{G}_{2}$ be a metric group with a metric $\mathrm{d}(\cdot, \cdot)$. Given $\varepsilon>0$, does there exist a $\delta>0$ such that if a function $\mathrm{h}: \mathrm{G}_{1} \rightarrow \mathrm{G}_{2}$ satisfies the inequality $\mathrm{d}(\mathrm{h}(\mathrm{xy}), \mathrm{h}(\mathrm{x}) \mathrm{h}(\mathrm{y}))<\delta$ for all $x, y \in \mathrm{G}_{1}$, then there exists a homomorphism $\mathrm{H}: \mathrm{G}_{1} \rightarrow \mathrm{G}_{2}$ with $\mathrm{d}(\mathrm{h}(\mathrm{x}), \mathrm{H}(\mathrm{x}))<\varepsilon$ for all $\mathrm{x} \in \mathrm{G}_{1}$ ?

In the following year, Hyers [6] gave a partial solution to the Ulam's problem for the case of approximate additive functions under the assumption that $\mathrm{G}_{1}$ and $\mathrm{G}_{2}$ are Banach spaces.

Considering this historical backgrounds, the additive Cauchy equation $f(x+y)=f(x)+f(y)$ on $\left(G_{1}, G_{2}\right)$ is said to be stable in the sense of Hyers and Ulam. This terminology is also applied to the

\footnotetext{
*Corresponding author

Email addresses: gkchoi@hongik.ac.kr (Ginkyu Choi), smjung@hongik.ac.kr (Soon-Mo Jung), yanghi2@hanmail.net (Yang-Hi Lee)

doi:10.22436/jnsa.010.08.42
} 
case of other functional equations. Moreover, Rassias [13] and Găvruţa [3] generalized the stability result of Hyers for the additive functional equation. The stability phenomenon proved by Rassias and Găvruţa is called the generalized Hyers-Ulam stability. For more detailed definitions of such terminologies, we refer the reader to $[2,7,8,9,10]$.

The functional equation

$$
f(x)-g(y)=(x-y) h(x+y),
$$

where $x, y \in \mathbb{R}$ (the set of reals), arises from the mean value theorem and characterizes polynomials of degree one or two. This functional equation was originally treated by Aczél in 1963 and also independently by Haruki (see [1, 5]). A generalization of the functional equation (1.2) was treated by Kannappan et al. [11] (see also [15, Theorem 2.5] or [1,5]). We summarize the result of Aczél regarding the equation (1.2) in the following theorem.

Theorem 1.1. Let $X$ be a field of characteristic different from 2 . The functions $f, g, h: X \rightarrow X$ satisfy the functional equation (1.1) for all $\mathrm{x}, \mathrm{y} \in \mathrm{X}$ if and only if there exist $\mathrm{a}, \mathrm{b}, \mathrm{c} \in \mathrm{X}$ such that

$$
f(x)=g(x)=a x^{2}+b x+c \text { and } h(x)=a x+b
$$

for all $x \in X$.

In Section 2 of this paper, we will prove the generalized Hyers-Ulam stability of the functional equation

$$
f(x)-g(y)=(x-y) h(s x+s y),
$$

where $s$ is a nonzero parameter. Even though the functional equation (1.2) seems to be the same one as (1.1), we investigate the equation (1.2) as a special form of the general equation

$$
f(x)-g(y)=(x-y) h(s x+t y) .
$$

The main result of this paper is a significant generalization of [10] as we shall see in Section 2. Section 3 is devoted to a characterization of quadratic polynomials.

\section{Hyers-Ulam stability of (1.2)}

In this section, let $X$ be a commutative normed algebra over $\mathrm{K}$ with a unit element $e$ (or a normed field of characteristic different from 2), where $K$ is either $\mathbb{R}$ or $\mathbb{C}$ (see [14, Chapter 10] for more detailed definition of normed algebra).

As usual, we assume that a commutative normed algebra $X$ has a sub-multiplicative norm $\|\cdot\|$, i.e., it satisfies the multiplicative inequality

$$
\|x y\| \leqslant\|x\|\|y\|,
$$

for all $x, y \in X$, which makes the multiplication a continuous operation in $X$.

For a given function $\varphi: X \times X \rightarrow[0, \infty)$, we will use the following notation

$$
\begin{aligned}
\Phi_{s}(x, y)= & \varphi\left(\frac{x+y}{2 s}, \frac{x-y}{2 s}\right)+2 \varphi\left(\frac{x+y}{2 s}, 0\right)+\varphi\left(\frac{x+y}{2 s}, \frac{y-x}{2 s}\right) \\
& +\varphi\left(\frac{y-x}{2 s}, \frac{x-y}{2 s}\right)+\varphi\left(\frac{y-x}{2 s}, 0\right)+2 \varphi\left(0, \frac{x-y}{2 s}\right)+\varphi\left(0, \frac{y-x}{2 s}\right)
\end{aligned}
$$

for all $x, y \in X$, where $s$ is a nonzero scalar parameter. Moreover, we assume that

$$
\lim _{n \rightarrow \infty} \frac{1}{2^{n}} \Phi_{s}\left(x, 2^{n} e\right)=0
$$

for all $x \in X$. 
For example, let $p$ and $\theta$ be positive real numbers with $0 \leqslant p<1$. If we temporarily define a function $\varphi: X \times X \rightarrow[0, \infty)$ by

$$
\varphi(x, y):=\theta\left(\|x\|^{p}+\|y\|^{p}\right)
$$

then

$$
\lim _{n \rightarrow \infty} \frac{1}{2^{n}} \Phi_{s}\left(x, 2^{n} e\right) \leqslant \lim _{n \rightarrow \infty} \frac{3 \theta}{2^{n+p-2}|s|^{p}}\left(\|x\|^{p}+2^{p n}\|e\|^{p}\right)=0 .
$$
2.3].

The proof of the following theorem is strongly based on the proof of [10, Theorem 2] or [15, Theorem

Theorem 2.1. Let $\mathrm{X}$ be a commutative normed algebra over $\mathrm{K}$ with a unit element e (or a normed field of characteristic different from 2 ), where $\mathrm{K}$ is either $\mathbb{R}$ or $\mathbb{C}$. Let $\mathrm{s}$ be a nonzero scalar parameter. Assume that a function $\varphi: X \times X \rightarrow[0, \infty)$ satisfies the condition (2.1). If functions $f, g, h: X \rightarrow X$ satisfy the inequality

$$
\|f(x)-g(y)-(x-y) h(s x+s y)\| \leqslant \varphi(x, y)
$$

for all $x, y \in X$, then there exist uniquely determined $a, b \in X$ such that

$$
\begin{aligned}
& \left\|f(x)-s a x^{2}-b x-g(0)\right\| \leqslant \varphi(x, 0), \\
& \left\|g(x)-s a x^{2}-b x-f(0)\right\| \leqslant \varphi(0, x), \\
& h(x)=a x+b
\end{aligned}
$$

for all $x \in X$.

Proof. If we put $y=0$ in (2.2), then we have

$$
\|f(x)-x h(s x)-g(0)\| \leqslant \varphi(x, 0)
$$

for each $x \in X$. Similarly, if we put $x=0$ in (2.2), then we get

$$
\|g(y)-y h(s y)-f(0)\| \leqslant \varphi(0, y)
$$

for all $y \in X$. Further, by (2.2), (2.4) and (2.5), we obtain

$$
\begin{aligned}
& \|x h(s x)-y h(s y)-(x-y) h(s x+s y)-f(0)+g(0)\| \\
& \quad \leqslant \quad\|f(x)-g(y)-(x-y) h(s x+s y)\|+\|x h(s x)-f(x)+g(0)\| \\
& \quad+\|g(y)-y h(s y)-f(0)\| \\
& \quad \leqslant \varphi(x, y)+\varphi(x, 0)+\varphi(0, y)
\end{aligned}
$$

for all $x, y \in X$. If we replace $x$ and $y$ in the last inequality with $\frac{1}{s} x$ and $\frac{1}{s} y$, respectively, then we have

$$
\begin{array}{r}
\|x h(x)-y h(y)-(x-y) h(x+y)-s f(0)+s g(0)\| \\
\leqslant|s| \varphi\left(\frac{x}{s}, \frac{y}{s}\right)+|s| \varphi\left(\frac{x}{s}, 0\right)+|s| \varphi\left(0, \frac{y}{s}\right)
\end{array}
$$

for all $x, y \in X$.

If $h$ satisfies inequality (2.6), so does $h+c$, where $c$ is an arbitrary element of $X$. Thus, we can assume that $h(0)=0$. If we replace $x$ by $-y$ in (2.6), then we get

$$
\begin{aligned}
& \|-y h(-y)-y h(y)-s f(0)+s g(0)\| \\
& \leqslant|s| \varphi\left(\frac{-y}{s}, \frac{y}{s}\right)+|s| \varphi\left(\frac{-y}{s}, 0\right)+|s| \varphi\left(0, \frac{y}{s}\right)
\end{aligned}
$$


for every $y \in X$. Moreover, if we replace $y$ by $-y$ in (2.6), then

$$
\begin{aligned}
& \| x h(x)+ y h(-y)-(x+y) h(x-y)-s f(0)+s g(0) \| \\
& \leqslant|s| \varphi\left(\frac{x}{s}, \frac{-y}{s}\right)+|s| \varphi\left(\frac{x}{s}, 0\right)+|s| \varphi\left(0, \frac{-y}{s}\right)
\end{aligned}
$$

for all $x, y \in X$. By (2.7) and (2.8), we further have

$$
\begin{aligned}
\| x h(x)- & y h(y)-(x+y) h(x-y)-2 s f(0)+2 s g(0) \| \\
\leqslant & \|x h(x)+y h(-y)-(x+y) h(x-y)-s f(0)+s g(0)\| \\
& +\|-y h(-y)-y h(y)-s f(0)+s g(0)\| \\
\leqslant & |s| \varphi\left(\frac{x}{s}, \frac{-y}{s}\right)+|s| \varphi\left(\frac{-y}{s}, \frac{y}{s}\right)+|s| \varphi\left(\frac{x}{s}, 0\right) \\
& +|s| \varphi\left(\frac{-y}{s}, 0\right)+|s| \varphi\left(0, \frac{y}{s}\right)+|s| \varphi\left(0, \frac{-y}{s}\right)
\end{aligned}
$$

for any $x, y \in X$.

On account of (2.6) and (2.9), we get

$$
\begin{aligned}
&\|(x+y) h(x-y)-(x-y) h(x+y)+s f(0)-s g(0)\| \\
& \leqslant\|-x h(x)+y h(y)+(x+y) h(x-y)+2 s f(0)-2 s g(0)\| \\
&+\|x h(x)-y h(y)-(x-y) h(x+y)-s f(0)+s g(0)\| \\
& \leqslant|s| \varphi\left(\frac{x}{s}, \frac{y}{s}\right)+2|s| \varphi\left(\frac{x}{s}, 0\right)+|s| \varphi\left(\frac{x}{s}, \frac{-y}{s}\right)+|s| \varphi\left(\frac{-y}{s}, \frac{y}{s}\right) \\
&+|s| \varphi\left(\frac{-y}{s}, 0\right)+2|s| \varphi\left(0, \frac{y}{s}\right)+|s| \varphi\left(0, \frac{-y}{s}\right)
\end{aligned}
$$

for every $x, y \in X$.

Substituting $u=x+y$ and $v=x-y$ in (2.10), we have

$$
\|u h(v)-v h(u)+s f(0)-s g(0)\| \leqslant|s| \Phi_{s}(u, v)
$$

for all $u, v \in X$. Furthermore, we obtain

$$
\|u h(v)-v h(u)\| \leqslant|s| \Phi_{s}(u, v)+|s||f(0)\|+|s|\| g(0) \|
$$

for any $u, v \in X$. Substituting $v=2^{n} e$ in (2.11), we have

$$
\left\|u h\left(2^{n} e\right)-2^{n} e h(u)\right\| \leqslant|s| \Phi_{s}\left(u, 2^{n} e\right)+|s||f(0)\|+|s| \mid g(0)\|
$$

and hence

$$
\left\|\frac{1}{2^{n}} u h\left(2^{n} e\right)-h(u)\right\| \leqslant \frac{|s|}{2^{n}}\left[\Phi_{s}\left(u, 2^{n} e\right)+\|f(0)\|+\|g(0)\|\right]
$$

for all $u \in X$ and $n \in \mathbb{N}$.

In view of (2.1), if we let $n \rightarrow \infty$ in (2.12), then we get

$$
h(u)=\lim _{n \rightarrow \infty} \frac{1}{2^{n}} u h\left(2^{n} e\right)
$$

for each $u \in X$. If we put $u=e$ in the last equality, then we obtain

$$
h(e)=\lim _{n \rightarrow \infty} \frac{1}{2^{n}} h\left(2^{n} e\right) .
$$


Since the multiplication is a continuous operation in $X$, we have

$$
h(x)=a x
$$

for all $x \in X$, where we set $a=h(e)$. If we do not assume $h(0)=0$, then we have

$$
h(x)=a x+b .
$$

In view of (2.4) and (2.13), we obtain

$$
\left\|f(x)-s a x^{2}-b x-g(0)\right\| \leqslant \varphi(x, 0)
$$

for any $x \in X$. Similarly, by (2.5) and (2.13), we have

$$
\left\|g(x)-s a x^{2}-b x-f(0)\right\| \leqslant \varphi(0, x)
$$

for all $x \in X$.

Finally, we will prove the uniqueness of $a$ and $b$. Assume that $a^{\prime}, b^{\prime} \in X$ satisfy the inequalities and equality in (2.3). By the second inequality of (2.3), we have

$$
\left\|s\left(a^{\prime}-a\right) x^{2}+\left(b^{\prime}-b\right) x\right\| \leqslant 2 \varphi(0, x)
$$

for all $x \in X$. If we set $x=\frac{2^{n-1}}{s} e$, then we obtain

$$
\left\|2^{n-1}\left(a^{\prime}-a\right) e^{2}+\left(b^{\prime}-b\right) e\right\| \leqslant \frac{4|s|}{2^{n}} \varphi\left(0, \frac{2^{n-1}}{s} e\right) \leqslant \frac{4|s|}{2^{n}} \Phi_{s}\left(0,2^{n} e\right)
$$

for all $n \in \mathbb{N}$. In view of (2.1), if we let $n \rightarrow \infty$ in the last inequality, then we have

$$
\lim _{n \rightarrow \infty} 2^{n-1}\left(a^{\prime}-a\right)=b-b^{\prime} .
$$

Hence, we conclude that $a^{\prime}-a=0$ and $b-b^{\prime}=0$, which completes our proof.

In Theorem 2.1, the difference between $f$ (or $g$ ) and a quadratic polynomial is bounded by $\varphi(x, 0)$ (or $\varphi(0, x)$ ), which is a significant improvement of [10, Theorem 2] even though the additional condition (2.1) is assumed in this paper. Moreover, $h$ is explicitly determined in this paper, while it was given approximately by using an inequality in [10, Theorem 2].

If $\varphi: X \times X \rightarrow[0, \infty)$ satisfies the additional condition

$$
\varphi(x, y) \geqslant \varphi(x, 0)+\varphi(0, y)+\varphi(0,0)
$$

as well as the condition (2.1), then the converse of Theorem 2.1 is also true. For example, if $X=\mathbb{R}$ and $\varphi(x, y)=\theta|x|^{p}|y|^{q}$ for some positive real numbers $p$, $q$ and $\theta$ with $0<p+q<1$, then all the conditions in (2.1) and (2.14) are satisfied.

Theorem 2.2. Let $\mathrm{X}$ be a commutative normed algebra over $\mathrm{K}$ with a unit element e (or a normed field of characteristic different from 2), where $\mathrm{K}$ is either $\mathbb{R}$ or $\mathbb{C}$. Let $\mathrm{s}$ be a nonzero scalar parameter. Assume that a function $\varphi: X \times X \rightarrow[0, \infty)$ satisfies the conditions (2.1) and (2.14) for all $x, y \in X$. Then functions $f, g, h: X \rightarrow X$ satisfy the inequality (2.2) for all $\mathrm{x}, \mathrm{y} \in \mathrm{X}$ if and only if there exist uniquely determined $\mathrm{a}, \mathrm{b} \in \mathrm{X}$ such that the inequalities and equality in (2.3) hold for all $\mathrm{x} \in \mathrm{X}$.

Proof. We only need to prove that if there exist uniquely determined $a, b \in X$ such that the inequalities and equality in (2.3) hold for all $x \in X$, then the functions $f, g, h: X \rightarrow X$ satisfy the inequality (2.2) for all $x, y \in X$. 
Indeed, it follows from (2.3) and (2.14) that

$$
\begin{aligned}
\|f(x)-g(y)-(x-y) h(s x+s y)\| \leqslant & \left\|f(x)-s a x^{2}-b x-g(0)\right\| \\
& +\left\|-g(y)+s a y^{2}+b y+f(0)\right\|+\|g(0)-f(0)\| \\
& \leqslant \varphi(x, 0)+\varphi(0, y)+\varphi(0,0) \\
& \leqslant \varphi(x, y)
\end{aligned}
$$

for all $x, y \in X$, i.e., the functions $f, g$, $h$ satisfy the inequality (2.2) for all $x, y \in X$. The proof is now complete. (1.2).

If we set $\varphi(x, y)=\varepsilon$ in Theorem 2.1, then we obtain the Hyers-Ulam stability of the functional equation

Corollary 2.3. Let $\mathrm{X}$ be a commutative normed algebra over $\mathrm{K}$ with a unit element e (or a normed field of characteristic different from 2 ), where $\mathrm{K}$ is either $\mathbb{R}$ or $\mathbb{C}$. For any previously given $\varepsilon>0$, if functions $\mathrm{f}, \mathrm{g}, \mathrm{h}: \mathrm{X} \rightarrow \mathrm{X}$ satisfy the inequality

$$
\|f(x)-g(y)-(x-y) h(s x+s y)\| \leqslant \varepsilon
$$

for all $\mathrm{x}, \mathrm{y} \in \mathrm{X}$ and for some nonzero scalar parameter $\mathrm{s}$, then there exist uniquely determined $\mathrm{a}, \mathrm{b} \in \mathrm{X}$ such that

$$
\begin{aligned}
& \left\|f(x)-s a x^{2}-b x-g(0)\right\| \leqslant \varepsilon, \\
& \left\|g(x)-s a x^{2}-b x-f(0)\right\| \leqslant \varepsilon, \\
& h(x)=a x+b
\end{aligned}
$$

for all $x \in X$.

In the following corollary, we prove the hyperstability of the functional equation

$$
x h(x)-y h(y)=(x-y) h(x+y),
$$

by using Theorem 2.1. That is, we will prove that every solution to a perturbed equation of (2.15) is just the solution to the exact equation (2.15). For more detailed definition of hyperstability, we refer the reader to $[4,12]$.

Corollary 2.4. Let $\mathrm{X}$ be a commutative normed algebra over $\mathrm{K}$ with a unit element e (or a normed field of characteristic different from 2), where $\mathrm{K}$ is either $\mathbf{R}$ or $\mathbf{C}$. Assume that a function $\varphi: X \times X \rightarrow[0, \infty)$ satisfies the condition (2.1) for $\mathrm{s}=1$. If any function $\mathrm{h}: \mathrm{X} \rightarrow \mathrm{X}$ satisfies the inequality

$$
\|x h(x)-y h(y)-(x-y) h(x+y)\| \leqslant \varphi(x, y)
$$

for all $x, y \in X$, then

$$
x h(x)-y h(y)=(x-y) h(x+y)
$$

for all $x, y \in X$.

Proof. If we put $s=1, f(x)=x h(x)$ and $g(y)=y h(y)$ for all $x, y \in X$, then the functions $f, g, h: X \rightarrow X$ satisfy inequality (2.2) for all $x, y \in X$. Therefore, the equality

$$
h(x)=a x+b
$$

holds for all $x \in X$. From the above equality, we get the desired equality

$$
x h(x)-y h(y)-(x-y) h(x+y)=x(a x+b)-y(a y+b)-(x-y)(a x+a y+b)=0
$$

for all $x, y \in X$. 


\section{A characterization of quadratic polynomials}

In this section, we introduce a characterization of polynomials of degree one or two as an application of Theorem 2.1.

Corollary 3.1. Let $\varphi: \mathbb{R} \times \mathbb{R} \rightarrow[0, \infty)$ be a function satisfying the condition (2.1) for a previously given nonzero parameter s. A differentiable function $\mathrm{f}: \mathbb{R} \rightarrow \mathbb{R}$ satisfies the inequality

$$
\left|f(x)-f(y)-(x-y) f^{\prime}(s x+s y)\right| \leqslant \varphi(x, y)
$$

for all $x, y \in \mathbb{R}$ if and only if there exist uniquely determined $a, b, c \in \mathbb{R}$ such that

$$
f(x)= \begin{cases}\frac{1}{2} a x^{2}+b x+c & \left(\text { for } s=\frac{1}{2}\right), \\ b x+c & \left(\text { for } s \notin\left\{0, \frac{1}{2}\right\}\right),\end{cases}
$$

for all $x \in \mathbb{R}$.

Proof. First, we assume that a differentiable function $f: \mathbb{R} \rightarrow \mathbb{R}$ satisfies the inequality (3.1) for all $x, y \in \mathbb{R}$. We set $g=f$ and $h=f^{\prime}$ in Theorem 2.1. Then there exist uniquely determined $a, b \in \mathbb{R}$ such that

$$
\left\{\begin{array}{l}
\left|f(x)-s a x^{2}-b x-f(0)\right| \leqslant \varphi(0, x), \\
f^{\prime}(x)=a x+b
\end{array}\right.
$$

for all $x \in \mathbb{R}$. By using the relations of (3.2), we have

$$
\left|\left(\frac{1}{2}-s\right) a\right| \leqslant \frac{1}{|x|^{2}} \varphi(0, x)
$$

for any $x \in \mathbb{R}$. If we set $x=\frac{2^{n-1}}{s}$ for $n \in \mathbb{N}$, then we get

$$
\left|\left(\frac{1}{2}-s\right) a\right| \leqslant \frac{s^{2}}{2^{n-2}} \frac{1}{2^{n}} \varphi\left(0, \frac{2^{n-1}}{s}\right) \leqslant \frac{s^{2}}{2^{n-2}} \frac{1}{2^{n}} \Phi_{s}\left(0,2^{n}\right)
$$

for each $n \in \mathbb{N}$. If we let $n \rightarrow \infty$ in the last inequality, then it follows from (2.1) that $\left|\left(\frac{1}{2}-s\right) a\right|=0$, i.e., $s=\frac{1}{2}$ or $a=0$. On account of (3.2), we see that

$$
f(x)=\frac{1}{2} a x^{2}+b x+c\left(\text { for } s=\frac{1}{2}\right) \quad \text { or } f(x)=b x+c \quad\left(\text { for } s \notin\left\{0, \frac{1}{2}\right\}\right)
$$

for all $x \in \mathbb{R}$.

Finally, we can easily verify the truth of the converse direction of our assertion. Hence, we omit the converse proof.

Szostok and Wassowicz proved in their paper [16] that if functions $f, F: \mathbb{R} \rightarrow \mathbb{R}$ satisfy the inequality

$$
\left|F(x)-F(y)-(x-y) f\left(\frac{x+y}{2}\right)\right| \leqslant \varepsilon
$$

for all $x, y \in \mathbb{R}$, then $f$ satisfies the equation

$$
G(x)-G(y)=(x-y) f\left(\frac{x+y}{2}\right)
$$

for all $x, y \in \mathbb{R}$, where we set $G(x)=x f\left(\frac{x}{2}\right)$. This superstability result of Szostok and Wassowicz is a special case of Corollary 3.1 with $s=\frac{1}{2}$. 


\section{Acknowledgment}

The authors are very grateful to the anonymous reviewers for their valuable remarks and suggestions that improved the first draft of this paper. Soon-Mo Jung was supported by Basic Science Research Program through the National Research Foundation of Korea (NRF) funded by the Ministry of Education (No. 2016R1D1A1B03931061).

\section{References}

[1] J. Aczél, A mean value property of the derivative of quadratic polynomials - without mean values and derivatives, Math. Mag., 58 (1985), 42-45. 1

[2] G. L. Forti, Hyers-Ulam stability of functional equations in several variables, Aequationes Math., 50 (1995), 143-190. 1

[3] P. Găvruţa, A generalization of the Hyers-Ulam-Rassias stability of approximately additive mappings, J. Math. Anal. Appl., 184 (1994), 431-436. 1

[4] E. Gselmann, Hyperstability of a functional equation, Acta Math. Hungar., 124 (2009), 179-188. 2

[5] S. Haruki, A property of quadratic polynomials, Amer. Math. Monthly, 86 (1979), 577-579. 1

[6] D. H. Hyers, On the stability of the linear functional equation, Proc. Natl. Acad. Sci. U. S. A., 27 (1941), 222-224. 1

[7] D. H. Hyers, G. Isac, T. M. Rassias, Stability of Functional Equations in Several Variables, Birkhäuser, Boston, (1998). 1

[8] D. H. Hyers, T. M. Rassias, Approximate homomorphisms, Aequationes Math., 44 (1992), 125-153. 1

[9] S.-M. Jung, Hyers-Ulam-Rassias Stability of Functional Equations in Nonlinear Analysis, Springer, New York, (2011). 1

[10] S.-M. Jung, P. K. Sahoo, On the stability of a mean value type functional equation, Demonstratio Math., 33 (2000), 793-796. 1, 1, 2, 2

[11] P. Kannappan, P. K. Sahoo, M. S. Jacobson, A characterization of low degree polynomials, Demonstratio Math., 28 (1995), 87-96. 1

[12] G. Maksa, Z. Páles, Hyperstability of a class of linear functional equations, Acta Math. Acad. Paedagog. Nyházi. (N.S.) 17 (2001), 107-112. 2

[13] T. M. Rassias, On the stability of the linear mapping in Banach spaces, Proc. Amer. Math. Soc., 72 (1978), 297-300. 1

[14] W. Rudin, Functional Analysis (2nd ed.), McGraw-Hill, New York, (1991). 2

[15] P. K. Sahoo, T. Riedel, Mean Value Theorems and Functional Equations, World Scientific, New Jersey, (1998). 1, 2

[16] T. Szostok, S. Wassowicz, On the stability of the equation stemming from Lagrange MVT, Appl. Math. Lett., 24 (2011), 541-544. 3

[17] S. M. Ulam, Problems in Modern Mathematics, Science Editions John Wiley and Sons, New York, (1964). 1 